\title{
THE FLOW OF A VISCOUS MHD FLUID*
}

\author{
$\mathrm{BY}$
}

\author{
O. P. CHANDNA AND M. R. GARG \\ University of Windsor, Ontario
}

1. Introduction. Martin [1] developed a new approach in the study of plane viscous flows of incompressible fluids. Following Martin [1], Nath and Chandna [2] studied plane viscous magnetohydrodynamic flows and derived the fundamental equations governing the flow in a new form, using streamlines and magnetic lines as the coordinate curves in a curvilinear system. These authors established the following results in case of orthogonal flows:

(i) if the streamlines are straight lines but not parallel, then they must be concurrent;

(ii) if the streamlines are involutes of a curve, then the streamlines must be concentric circles.

The above approach is valid if magnetic lines and streamlines do not coincide and thereby excludes aligned flows from consideration. Throughout this paper we study plane, steady flows of a viscous incompressible fluid of infinite electrical conductivity for which the magnetic field vector lies in the flow plane and makes a constant angle $\theta$ with the velocity vector, including the case of aligned flows. We introduce curvilinear coordinates $\phi, \psi$ in the physical plane in which the coordinate lines $\psi=$ constant are the streamlines and the lines $\phi=$ constant are left arbitrary. For instance, orthogonal trajectories of streamlines, or in the case of non-aligned flow, magnetic lines can be taken as the lines $\phi=$ constant. For the flows under study we establish the following:

(i) If the streamlines are straight lines then they must be concurrent or parallel.

(ii) If the streamlines are involutes of a curve, then they must be concentric circles.

(iii) If the streamlines $\psi=$ constant and their orthogonal trajectories $\phi=$ constant generate an isometric net, then the streamlines are restricted to parallel straight lines, concurrent lines, concentric circles or logarithmic spirals if $\theta \neq \pi / 2$. This result was first established by Hamel [3] and later proved using new approach by Martin [1] for non-MHD flows.

2. Flow equations. The steady, plane flow of a viscous, incompressible fluid of infinite electrical conductivity is governed by the following system of equations:

$$
\begin{gathered}
\partial \rho u / \partial x+\partial \rho v / \partial y=0 \\
\rho\left(u \frac{\partial u}{\partial x}+v \frac{\partial u}{\partial y}\right)+\frac{\partial p}{\partial x}=\eta\left(\frac{\partial^{2} u}{\partial x^{2}}+\frac{\partial^{2} u}{\partial y}\right)-\mu H_{2}\left(\frac{\partial H_{2}}{\partial x}-\frac{\partial H_{1}}{\partial y}\right) \\
\rho\left(u \frac{\partial v}{\partial x}+v \frac{\partial v}{\partial y}\right)+\frac{\partial p}{\partial y}=\eta\left(\frac{\partial^{2} v}{\partial x^{2}}+\frac{\partial^{2} v}{\partial y}\right)+\mu H_{1}\left(\frac{\partial H_{2}}{\partial x}-\frac{\partial H_{1}}{\partial y}\right)
\end{gathered}
$$

*Received January 20,1975; revised version received May 29, 1975. The authors wish to thank the referee for his comments and the National Research Council of Canada for financial support. 


$$
\begin{gathered}
u H_{2}-v H_{1}=k, \\
\partial H_{1} / \partial x+\partial H_{2} / \partial y=0,
\end{gathered}
$$

where $u, v$ are the velocity components, $H_{1}, H_{2}$ the components of magnetic field vector $\mathbf{H}, p$ the pressure function, $\eta$ the constant coefficient of viscosity, $\rho$ the constant density, $\mu$ the constant magnetic permeability and $k$ an arbitrary constant which is zero for the aligned flows and non-zero in the case of non-aligned flows.

We define the following functions:

$$
\begin{gathered}
\omega=\frac{\partial v}{\partial x}-\frac{\partial u}{\partial y}, \quad Q=\frac{\partial H_{2}}{\partial x}-\frac{\partial H_{1}}{\partial y}, \\
h=\frac{1}{2} \rho q^{2}+p,
\end{gathered}
$$

wherein $q=\left(u^{2}+v^{2}\right)^{1 / 2}$.

In terms of these functions, the system of equations (1)-(5) is replaced by the following system:

$$
\begin{gathered}
\partial \rho u / \partial x+\partial \rho v / \partial y=0, \\
\eta(\partial \omega / \partial y)-\rho \omega v+\mu Q H_{2}=-(\partial h / \partial x), \\
\eta(\partial \omega / \partial x)-\rho \omega u+\mu Q H_{1}=\partial h / \partial y, \\
u H_{2}-v H_{1}=k, \\
\partial H_{1} / \partial x+\partial H_{2} / \partial y=0, \\
\partial v / \partial x-\partial u / \partial y=\omega \\
\partial H_{2} / \partial x-\partial H_{1} / \partial y=Q
\end{gathered}
$$

of seven equations in seven unknowns $u, v, H_{1}, H_{2}, \omega, Q$ and $h$ as functions of $x, y$. The advantage of this system over the original system is that the order of partial differential equations has decreased from two to one.

Eq. (7) implies the existence of a stream function $\psi(x, y)$ such that

$$
\partial \psi / \partial x=-\rho v, \partial \psi / \partial y=\rho u
$$

and $\psi(x, y)=$ constant defines the family of streamlines. Let us take $\phi(x, y)=$ constant to be some arbitrary family of curves such that it gencrates with $\psi(x, y)=$ constant a curvilinear net $(\phi, \psi)$ in the physical plane.

Let

$$
x=x(\phi, \psi), \quad y=y(\phi, \psi)
$$

define the curvilinear net in $(x, y)$-plane and let the squared element of arc length along any curve be

$$
d s^{2}=E(\phi, \psi) d \phi^{2}+2 F(\phi, \psi) d \phi d \psi+G(\phi, \psi) d \psi^{2}
$$

where

$$
\begin{gathered}
E=\left(\frac{\partial x}{\partial \phi}\right)^{2}+\left(\frac{\partial y}{\partial \phi}\right)^{2}, \quad F=\left(\frac{\partial x}{\partial \phi}\right)\left(\frac{\partial x}{\partial \psi}\right)+\left(\frac{\partial y}{\partial \phi}\right)\left(\frac{\partial y}{\partial \psi}\right) \\
G=\left(\frac{\partial x}{\partial \psi}\right)^{2}+\left(\frac{\partial y}{\partial \psi}\right)^{2} .
\end{gathered}
$$


Eq. (15) can be solved to obtain

$$
\phi=\phi(x, y), \quad \psi=\psi(x, y)
$$

such that

$$
\frac{\partial x}{\partial \phi}=J \frac{\partial \psi}{\partial y}, \quad \frac{\partial x}{\partial \psi}=-J \frac{\partial \phi}{\partial y}, \quad \frac{\partial y}{\partial \phi}=-J \frac{\partial \psi}{\partial x}, \quad \frac{\partial y}{\partial \psi}=J \frac{\partial \phi}{\partial x}
$$

provided that $0<|J|<\infty$, where $J$ is the transformation Jacobian, and

$$
J=\left(\frac{\partial x}{\partial \phi}\right)\left(\frac{\partial y}{\partial \psi}\right)-\left(\frac{\partial x}{\partial \psi}\right)\left(\frac{\partial y}{\partial \phi}\right) .
$$

Letting $\alpha$ denote the local angle of inclination of the tangent to the coordinate line $\psi=$ constant, directed in the sense of increasing $\phi$, we have, from differential geometry, the following results $[4]$ :

$$
\begin{gathered}
J= \pm W, \quad W=\left(E\left(i-F^{2}\right)^{1 / 2},\right. \\
\frac{\partial x}{\partial \phi}=\sqrt{ } E \cos \alpha, \quad \frac{\partial y}{\partial \phi}=\sqrt{ } E \sin \alpha, \\
\frac{\partial x}{\partial \psi}=\frac{F}{\sqrt{ } E} \cos \alpha-\frac{J}{\sqrt{ } E} \sin \alpha, \quad \frac{\partial y}{\partial \psi}=\frac{F}{\sqrt{ } E} \sin \alpha+\frac{J}{\sqrt{ }{ }^{\prime}} \cos \alpha, \\
\frac{\partial \alpha}{\partial \phi}=\frac{J}{E} \Gamma_{11}{ }^{2}, \quad \frac{\partial \alpha}{\partial \psi}=\frac{J}{E} \Gamma_{12}{ }^{2},
\end{gathered}
$$

where

$$
\begin{aligned}
& \Gamma_{11}^{2}=\frac{1}{2 W^{2}}\left(-F \frac{\partial E}{\partial \phi}+2 E \frac{\partial F}{\partial \phi}-E \frac{\partial E}{\partial \psi}\right), \\
& \Gamma_{12}^{2}=\frac{1}{2 W^{2}}\left(E \frac{\partial G}{\partial \phi}-F \frac{\partial E}{\partial \psi}\right) .
\end{aligned}
$$

The three functions $E, F, G$ of $\phi, \psi$ satisfy the Gauss equation:

$$
K=\frac{1}{W}\left[\frac{\partial}{\partial \psi}\left(\frac{W}{E} \Gamma_{11}^{2}\right)-\frac{\partial}{\partial \phi}\left(\frac{W}{E} \Gamma_{12}^{2}\right)\right]=0
$$

where $K$ is the Gaussian curvature.

We shall now study only those flows in which the magnetic field vector $\mathbf{H}$ makes a constant angle $\theta$ with the velocity vector $\mathrm{V}$. Aligned flows are treated as special cases of such flows.

In the case of non-aligned flows with non-zero constant angle $\langle\mathbf{V}, \mathbf{H}\rangle=\theta$, (10) implies that

$$
H q \sin \theta=k, \quad k \neq 0
$$

where $H=|\mathbf{H}|$.

In the case of aligned flows, we have

$$
\mathbf{H}=f \mathbf{V}
$$


where $f$ is some arbitrary scalar function. In view of (7) and (11), we find that $\int$ is constant along each individual streamline, i.e.

$$
\mathrm{V} \cdot \operatorname{grad} f=0 .
$$

Consequently, in case of aligned flows, we have

$$
H=|f(\psi)| q .
$$

Next, we transform the flow equations into a new form with $\phi, \psi$ as independent variables.

3. New form for the fundamental equations. Equations of continuity and vorticity: Martin [1] has shown that the equation of continuity (7) implies that the fluid flows along the streamlines towards higher or lower parameter values of $\phi$ accordingly as $J$ is positive or negative and that

$$
W \rho q=\sqrt{ } E, \quad u+i v=\frac{\sqrt{ } E}{\rho J} \exp (i \alpha) .
$$

He has also proven that

$$
\omega=\frac{1}{\rho W}\left[\frac{\partial}{\partial \phi}\left(\frac{F}{W}\right)-\frac{\partial}{\partial \psi}\left(\frac{E}{W}\right)\right] .
$$

Solenoidal condition on $H: \quad \mathrm{H}$ makes an angle $\theta+\alpha$ or $\theta+\alpha-\pi$ with the $x$-axis according as fluid flows along the streamlines towards higher or lower parameter values $\phi$ and so we have

$$
H_{1}= \pm H \cos (\theta+\alpha), \quad H_{2}= \pm H \sin (\theta+\alpha)
$$

where the positive or negative sign is taken according as $J$ is positive or negative.

Using (17) in (11), it follows that

$$
\left(\frac{\partial H_{1}}{\partial \phi} \cdot \frac{\partial y}{\partial \psi}-\frac{\partial H_{1}}{\partial \psi} \cdot \frac{\partial y}{\partial \phi}\right)-\left(\frac{\partial H_{2}}{\partial \phi} \cdot \frac{\partial x}{\partial \psi}-\frac{\partial H_{2}}{\partial \psi} \cdot \frac{\partial x}{\partial \phi}\right)=0 .
$$

Employing (18) and (24) in this equation, we have

$$
\begin{aligned}
& \frac{\partial H}{\partial \phi}(J \cos \theta-F \sin \theta)+\frac{\partial H}{\partial \psi} E \sin \theta-H \frac{J}{E} \mathrm{\Gamma}_{11}{ }^{2}(F \cos \theta+J \sin \theta) \\
& +H \frac{J}{E} \Gamma_{12}{ }^{2} E \cos \theta=0 .
\end{aligned}
$$

The function $Q:$ By the definition of $Q$ and (17) we have

$$
J Q=\left(\frac{\partial H_{2}}{\partial \phi} \cdot \frac{\partial y}{\partial \psi}-\frac{\partial H_{2}}{\partial \psi} \cdot \frac{\partial y}{\partial \phi}\right)+\left(\frac{\partial H_{1}}{\partial \phi} \cdot \frac{\partial x}{\partial \psi}-\frac{\partial H_{1}}{\partial \psi} \cdot \frac{\partial x}{\partial \phi}\right) .
$$

Using (18) and (24) in this equation, we get

$$
\begin{aligned}
\sqrt{ } E W Q=\frac{\partial H}{\partial \phi}(F \cos \theta+ & J \sin \theta)-\frac{\partial H}{\partial \psi} E \cos \theta \\
& -H \frac{J}{E} \Gamma_{11}{ }^{2}\left(F^{\prime} \sin \theta-J \cos \theta\right)+H \frac{J}{E} \Gamma_{12}{ }^{2} E \sin \theta .
\end{aligned}
$$


Momentum equations: Using (17) and (18) in (8), we have

$$
\eta\left[-\frac{\partial \omega}{\partial \phi} \cdot \frac{\partial x}{\partial \psi}+\frac{\partial \omega}{\partial \psi} \cdot \frac{\partial x}{\partial \phi}\right]-\omega \frac{\partial y}{\partial \phi}+\mu W Q H \sin (\theta+\alpha)=-\left(\frac{\partial h}{\partial \phi} \cdot \frac{\partial y}{\partial \psi}-\frac{\partial h}{\partial \psi} \frac{\partial y}{\partial \phi}\right) .
$$

Likewise, (9) takes the form

$$
\eta\left[\frac{\partial \omega}{\partial \phi} \cdot \frac{\partial y}{\partial \psi}-\frac{\partial \omega}{\partial \psi} \cdot \frac{\partial y}{\partial \phi}\right]-\omega \frac{\partial x}{\partial \phi}+\mu W Q H \cos (\theta+\alpha)=\left(-\frac{\partial h}{\partial \phi} \cdot \frac{\partial x}{\partial \psi}+\frac{\partial h}{\partial \psi} \cdot \frac{\partial x}{\partial \phi}\right) .
$$

Multiplying (27) by $\partial y / \partial \phi$ and (28) by $\partial x / \partial \phi$ and adding, we get

$$
\eta J \frac{\partial \omega}{\partial \phi}-\omega E+\mu W Q H \sqrt{ } E \cos \theta=-F \frac{\partial h}{\partial \phi}+E \frac{\partial h}{\partial \psi} .
$$

Likewise, multiplying (27) by $\partial y / \partial \psi$ and (28) by $\partial x / \partial \psi$ and adding, we have

$$
\eta J \frac{\partial \omega}{\partial \psi}-\omega F+\mu W Q H\left(\frac{F}{\sqrt{ } E} \cos \theta+\frac{J}{\sqrt{ } E} \sin \theta\right)=-G \frac{\partial h}{\partial \phi}+F \frac{\partial h}{\partial \psi} .
$$

Summing up, we have:

Theorem 1: If the streamlines $\psi=$ constant and an arbitrarily chosen family of curves $\phi=$ constant generate a curvilinear net $(\phi, \psi)$ in the physical plane of fluid flow under study, the system of seven partial differential equations (7)-(13) involving seven unknowns $u, v, H_{1}, H_{2}, \omega, Q$ and $h$ is replaced by the new system

$$
\begin{gathered}
\eta J \frac{\partial \omega}{\partial \phi}-E \omega+\mu W Q H \sqrt{ } E \cos \theta=E \frac{\partial h}{\partial \psi}-F \frac{\partial h}{\partial \phi}, \\
\eta J \frac{\partial \omega}{\partial \psi}-F \omega+\mu W Q H\left(\frac{F}{\sqrt{ } E} \cos \theta+\frac{J}{\sqrt{ } E} \sin \theta\right)=F \frac{\partial h}{\partial \psi}-G \frac{\partial h}{\partial \phi}, \\
\frac{\partial}{\partial \psi}\left(\frac{W}{E} \Gamma_{11}^{2}\right)-\frac{\partial}{\partial \phi}\left(\frac{W}{E} \Gamma_{12}^{2}\right)=0, \quad \omega=\frac{1}{\rho W}\left[\frac{\partial}{\partial \phi}\left(\frac{F}{W}\right)-\frac{\partial}{\partial \psi}\left(\frac{E}{W}\right)\right],
\end{gathered}
$$

$\frac{\partial H}{\partial \phi}(J \cos \theta-F \sin \theta)+\frac{\partial H}{\partial \psi} E \sin \theta$

$$
-H \frac{J}{E} \Gamma_{11}^{2}(F \cos \theta+J \sin \theta)+H \frac{J}{E} \Gamma_{12}{ }^{2} E \cos \theta=0,
$$

$\sqrt{ } E W Q=\frac{\partial H}{\partial \phi}(F \cos \theta+J \sin \theta)-\frac{\partial H}{\partial \psi} E \cos \theta$

$$
\left.-H \frac{J}{E} \Gamma_{11}{ }^{2}(F \sin \theta-J \cos \theta)+H \frac{J}{E} \Gamma_{12}^{2} E \sin \theta\right)
$$

of six partial differential equations in seven unknowns $E, F, G, \omega, Q, H$ and $h$. Given a solution

$$
\begin{array}{lll}
E=E(\phi, \psi), & F=F(\phi, \psi), & G=G(\phi, \psi), \quad h=h(\phi, \psi) \\
\omega=\omega(\phi, \psi), & H=H(\phi, \psi), & Q=Q(\phi, \psi)
\end{array}
$$


of the system (31), we can find $x, y$ as functions of $\phi, \psi$ from

$$
z=\int \frac{\exp (i \alpha)}{\sqrt{ } E}\{E d \phi+(F+i J) d \psi\}
$$

where

$$
\alpha=\int \frac{J}{E}\left\{\Gamma_{11}^{2} d \phi+\Gamma_{12}^{2} d \psi\right\} .
$$

$E, F, G, h, \omega, H$ and $Q$ may now be obtained as functions of $x, y$ and thus $H_{1}, H_{2}, u, v$ and $p$ are obtained as functions of $x, y$ from:

$$
\begin{gathered}
H_{1}+i H_{2}=\left(\frac{H}{J}\right) \exp [i(\theta+\alpha)], \\
u+i v=\frac{\sqrt{ } E^{\prime}}{J} \exp (i \alpha), \quad p=h-\frac{E}{2 \rho W^{2}} .
\end{gathered}
$$

The system (31) is an underdetermined system as there are seven unknowns and only six equations. It can be made a determinate system in several ways. We consider one of the methods of making above system determinate. In this method the orthogonal trajectories of the streamlines $\psi=$ constant are chosen as coordinate lines $\phi=$ constant. In this case $F=0$ and the momentum equations (29), (30) become

$$
\begin{gathered}
\eta \frac{J}{E} \frac{\partial \omega}{\partial \phi}-\omega+\mu Q H \sqrt{ }\left(i \cos \theta=\frac{\partial h}{\partial \psi},\right. \\
-\eta \frac{J}{C_{i}} \frac{\partial \omega}{\partial \psi}-\mu Q H-\frac{J}{\sqrt{ }(\dot{r}} \sin \theta=\frac{\partial h}{\partial \phi} .
\end{gathered}
$$

Using the integrability condition $\partial^{2} h / \partial \phi \partial \psi=\partial^{2} h / \partial \psi \partial \phi$, we get

$$
\begin{aligned}
\eta\left[\frac{\partial}{\partial \phi}\left(\frac{J}{E} \frac{\partial \omega}{\partial \phi}\right)+\frac{\partial}{\partial \psi}\left(\frac{J}{G_{i}} \frac{\partial \omega}{\partial \psi}\right)\right] & -\frac{\partial \omega}{\partial \phi} \\
& +\mu\left[\frac{\partial}{\partial \phi}\left(Q H \sqrt{ } G_{i}\right) \cos \theta+\frac{\partial}{\partial \psi}\left(Q H \frac{J}{\sqrt{ } G^{(}}\right) \sin \theta\right]=0
\end{aligned}
$$

Therefore, we state:

Conollary 1: If streamlines $\psi=$ constant and their orthogonal trajectories $\phi=$ constant are taken as $(\phi, \psi)$ net in the physical plane, the fluid flow under study is represented by the system

$$
\begin{aligned}
& \eta\left[\frac{\partial}{\partial \phi}\left(\frac{J}{E} \frac{\partial \omega}{\partial \phi}\right)+\frac{\partial}{\partial \psi}\left(\frac{J}{(i} \frac{\partial \omega}{\partial \psi}\right)\right]-\frac{\partial \omega}{\partial \phi}+\mu\left[\frac{\partial}{\partial \phi}\left(Q H \sqrt{ }(i) \cos \theta+\frac{\partial}{\partial \psi}\left(Q H \frac{J}{\sqrt{(i}}\right) \sin \theta\right]=0\right. \\
& \frac{\partial}{\partial \psi}\left(\frac{1}{\left(E(i)^{1 / 2}\right.} \frac{\partial E}{\partial \psi}\right)+\frac{\partial}{\partial \phi}\left(\frac{1}{\left(E(i)^{1 / 2}\right.} \frac{\partial G}{\partial \phi}\right)=0 \\
& \omega=-\frac{1}{\rho W} \frac{\partial}{\partial \psi}\left(\frac{E}{W}\right)
\end{aligned}
$$




$$
\begin{gathered}
J \cos \theta \frac{\partial H}{\partial \phi}+E \sin \theta \frac{\partial H}{\partial \psi}+\frac{1}{2} H \sin \theta \frac{\partial E}{\partial \psi}+\frac{1}{2} H \frac{J}{G} \cos \theta \frac{\partial G}{\partial \phi}=0, \\
\sqrt{ } E W Q=J \sin \theta \frac{\partial H}{\partial \phi}-E \cos \theta \frac{\partial H}{\partial \psi}-\frac{1}{2} H \cos \theta \frac{\partial E}{\partial \psi}+\frac{1}{2} H \frac{J}{G} \sin \theta \frac{\partial G}{\partial \phi}
\end{gathered}
$$

of five partial differential equations in five unknowns $E, G, \omega, H$ and $Q$.

4. Straight streamlines. In this section we inquire what plane flow patterns are possible when the streamlines are straight lines. For electrically non-conducting viscous flows this result has been studied by Martin [1], and streamline pattern is either parallel or concurrent lines. Nath and Chandna [2] extended this result to electrically conducting fluids for orthogonal flows.

Now we investigate the same problem for constantly inclined velocity and magnetic fields. In order to approach the answer, we assume that the streamlines are non-parallel straight lines enveloping a curve $C$. Taking the tangent lines to $C$ and their orthogonal trajectories (the involutes of $C$ ) as a system of orthogonal curvilinear coordinates, the squared element of arc length is given by [4]

$$
d s^{2}=d \xi^{2}+(\xi-\sigma) \kappa^{2} d \sigma^{2}
$$

where $\sigma$ denotes the arc length, $\kappa$ the curvature of $C$ and $\xi$ the parameter constant along each individual involute. If $\nu$ denotes the angle of elevation of the tangent line to $C$, we have $d \nu / d \sigma=\kappa$ and (35) becomes

$$
d s^{2}=d \xi^{2}+(\xi-\sigma) d \nu^{2} \text { where } \sigma=\sigma(\nu) .
$$

In this coordinate system the coordinate curves $\xi=$ constant and $\nu=$ constant are respectively the involutes of $C$ and the tangent lines of $C$.

We now investigate the flows for which

$$
\phi=\phi(\xi), \psi=\psi(\nu) .
$$

Using (37) in (16), we get

$$
d s^{2}=E \phi^{\prime 2} d \xi^{2}+2 F \phi^{\prime} \psi^{\prime} d \xi d \nu+G \psi^{\prime 2} d \nu^{2} .
$$

Comparing (38) with (36), we obtain

$$
E^{\prime}=\frac{1}{\left[\phi^{\prime}(\xi)\right]^{2}}, \quad F=0, \quad G=\left[\frac{\xi-\sigma(\nu)}{\psi^{\prime}(\nu)}\right]^{2} .
$$

We assume that $J>0$.

Using (39) and introducing $\xi, \nu$ as new variables in the Gauss equation (20), we find that it is automatically satisfied. Substituting $E, F, G$ from (39) into (34), we have

$$
\begin{gathered}
\eta \frac{\partial}{\partial \xi}\left[(\xi-\sigma) \frac{\partial \omega}{\partial \xi}\right]+\eta \frac{\partial}{\partial \nu}\left[\frac{1}{(\xi-\sigma)} \frac{\partial \omega}{\partial \nu}\right]-\psi^{\prime} \frac{\partial \omega}{\partial \xi} \\
+\mu \frac{\partial}{\partial \xi}[Q H(\xi-\sigma)] \cos \theta+\mu \frac{\partial}{\partial \nu}(Q H) \sin \theta=0 \\
\omega=-\frac{1}{\rho(\xi-\sigma)} \frac{\partial}{\partial \nu}\left(\frac{\psi^{\prime}}{\xi-\sigma}\right)
\end{gathered}
$$




$$
\begin{gathered}
\frac{\partial H}{\partial \xi}(\xi-\sigma) \cos \theta+\frac{\partial H}{\partial \nu} \sin \theta+H \cos \theta=0, \\
(\xi-\sigma) Q=\frac{\partial H}{\partial \xi}(\xi-\sigma) \sin \theta-\frac{\partial H}{\partial \nu} \cos \theta+H \sin \theta .
\end{gathered}
$$

Eqs. (40)-(43) are valid for both aligned and non-aligned flows. However, for the nonaligned case $q H \sin \theta=k \neq 0$, as given in (21), is the additional restriction. This restriction is not valid for aligned flows and, therefore, we use (40) to (43) with $\theta=0$ for such flows.

First we consider the non-aligned flows. Using (21), (41), (42) and (43) in (40), we obtain the condition

$$
\begin{aligned}
15 \eta{\sigma^{\prime}}^{3} \psi^{\prime} & +(\xi-\sigma)\left[15 \eta{\sigma^{\prime}}^{2} \psi^{\prime \prime}+10 \eta \sigma^{\prime} \sigma^{\prime \prime} \psi^{\prime}\right]+(\xi-\sigma)^{2}\left[9 \eta \psi^{\prime} \sigma^{\prime}+4 \eta \sigma^{\prime \prime} \psi^{\prime \prime}\right. \\
& \left.+6 \eta \sigma^{\prime} \psi^{\prime \prime \prime}+\eta \sigma^{\prime \prime \prime} \psi^{\prime}+3 \psi^{\prime 2} \sigma^{\prime}\right]+(\xi-\sigma)^{3}\left[4 \eta \psi^{\prime \prime}+\eta \psi^{(i v)}+2 \psi^{\prime} \psi^{\prime \prime}\right] \\
& +(\xi-\sigma)^{6} \frac{\mu k^{2}}{\sin ^{2} \theta} \frac{\sigma^{\prime}}{\psi^{2}}+(\xi-\sigma)^{7} \frac{2 \mu k^{2}}{\sin ^{2} \theta} \frac{\psi^{\prime \prime}}{\psi^{3}} \equiv 0 .
\end{aligned}
$$

Since $\xi, \nu$ are independent variables, for the relation (44) to hold identically, it must hold on the curve $C$ which appears as $\xi=\sigma(\nu)$, and consequently

$$
15 \eta{\sigma^{\prime}}^{3} \psi^{\prime}=0 .
$$

This implies that $\sigma^{\prime}=0$ as $\psi^{\prime}$ cannot vanish identically. Therefore, the radius of curvature $R\left(=\sigma^{\prime}(\nu)\right)$ of $C$ vanishes identically and streamlines are concurrent lines. Now we consider aligned flows. Eqs. (40)-(43) reduce to the following form:

$$
\begin{gathered}
\eta \frac{\partial}{\partial \xi}\left[(\xi-\sigma) \frac{\partial \omega}{\partial \xi}\right]+\eta \frac{\partial}{\partial \nu}\left[\frac{1}{(\xi-\sigma)} \frac{\partial \omega}{\partial \nu}\right]-\psi^{\prime} \frac{\partial \omega}{\partial \xi}+\mu \frac{\partial}{\partial \xi}[Q H(\xi-\sigma)]=0 \\
\omega=-\frac{1}{\rho(\xi-\sigma)} \frac{\partial}{\partial \nu}\left[\frac{\psi^{\prime}}{\xi-\sigma}\right] \\
\frac{\partial}{\partial \xi}[H(\xi-\sigma)]=0 \\
(\xi-\sigma) Q=-\partial H / \partial \nu
\end{gathered}
$$

Eq. (47) implies that

$$
H=F(\nu) /(\xi-\sigma)
$$

where $F(\nu)$ is an arbitrary function of $\nu$. From (45), (46), (48) and (49), we get

$$
\begin{aligned}
15 \eta \sigma^{\prime 3} \psi^{\prime}+ & (\xi-\sigma)\left(15 \eta \sigma^{\prime 2} \psi^{\prime \prime}+10 \sigma^{\prime} \sigma^{\prime \prime} \psi^{\prime}\right) \\
& +(\xi-\sigma)^{2}\left[9 \eta \sigma^{\prime} \psi^{\prime}+4 \eta \sigma^{\prime \prime} \psi^{\prime \prime}+6 \eta \sigma^{\prime} \psi^{\prime \prime \prime}+\eta \sigma^{\prime \prime \prime} \psi^{\prime}+3 \sigma^{\prime} \psi^{\prime 2}-3 \mu \sigma^{\prime} F^{2}(\nu)\right] \\
& +(\xi-\sigma)^{3}\left[4 \eta \psi^{\prime \prime}+\eta \psi^{(i v)}+2 \psi^{\prime} \psi^{\prime \prime}-2 \mu F(\nu) F^{\prime}(\nu)\right] \equiv 0 .
\end{aligned}
$$

By the same argument as used earlier, $\sigma^{\prime} \equiv 0$ and so the radius of curvature of $C$ is zero and streamlines are concurrent. Summing up, we have:

Theorem 2: If the streamlines in steady, plane flow of a viscous incompressible fluid of finite electrical conductivity are straight lines, they must be concurrent or parallel. 
5. Flows in which streamlines are involutes of a curve $C$. In this section we investigate flows in which streamlines $\psi=$ constant are involutes of a curve $C$. Martin [1] studied such steady, plane flows of viscous incompressible fluids and found that streamlines must be concentric circles. Nath and Chandna [2] extended this result to orthogonal magnetohydrodynamic flows.

As in Sec. 4, we take the curvilinear coordinate system $(\xi, \nu)$ where coordinate curves $\xi=$ constant and $\nu=$ constant are the involutes and tangent lines of $C$ respectively. We now seek those flows for which

$$
\phi=\phi(\nu), \quad \psi=\psi(\xi) .
$$

The squared element of arc length is

$$
d s^{2}=d \xi^{2}+(\xi-\sigma) d \nu^{2} .
$$

Using (50) in (16) and comparing with (51), we get

$$
E=\left(\frac{\xi-\sigma}{\phi^{\prime}}\right)^{2}, \quad F=0, \quad G=\left(\frac{1}{\psi^{\prime}}\right)^{2}
$$

We assume $J=(\xi-\sigma) / \phi^{\prime} \psi^{\prime}>0$. The Gauss equation is again automatically satisfied.

With the introduction of $E, F, G$ from (52) in (34), we obtain

$$
\begin{gathered}
\eta\left[\frac{\partial}{\partial \nu}\left\{\frac{1}{(\xi-\sigma)} \frac{\partial \omega}{\partial \nu}\right\}+\frac{\partial}{\partial \xi}\left\{(\xi-\sigma) \frac{\partial \omega}{\partial \xi}\right\}\right]-\psi^{\prime} \frac{\partial \omega}{\partial v} \\
+\mu\left[\frac{\partial}{\partial \nu}(Q H) \cos \theta+\frac{\partial}{\partial \xi}\{Q H(\xi-\sigma)\} \sin \theta\right]=0 \\
\omega=-\frac{1}{\rho(\xi-\sigma)} \frac{\partial}{\partial \xi}\left[(\xi-\sigma) \psi^{\prime}\right] \\
\frac{\partial H}{\partial \nu} \cos \theta+\frac{\partial H}{\partial \xi}(\xi-\sigma) \sin \theta+H \sin \theta=0 \\
(\xi-\sigma) Q=\frac{\partial H}{\partial \nu} \sin \theta-\frac{\partial H}{\partial \xi}(\xi-\sigma) \cos \theta-H \cos \theta
\end{gathered}
$$

Eqs. (53)-(56) are valid for non-aligned as well as aligned flows. First we consider the non-aligned flows. Using (21), (46), (55) and (56) in (53), we obtain the condition

$$
\begin{array}{r}
3 \eta \psi^{\prime} \sigma^{\prime 2}+\eta \psi^{\prime} \sigma^{\prime \prime}(\xi-\sigma)+\left(\eta \psi^{\prime}-\sigma^{\prime} \psi^{\prime 2}\right)(\xi-\sigma)^{2}-\eta \psi^{\prime \prime}(\xi-\sigma)^{3}+2 \eta \psi^{\prime \prime \prime}(\xi-\sigma)^{4} \\
+\eta \psi^{(i v)}(\xi-\sigma)^{5} \equiv 0 .
\end{array}
$$

For the relation (57) to hold identically, it must hold on the curve $C$ given by $\xi=\sigma(\nu)$ and consequently $\sigma^{\prime} \equiv 0$ since $\psi^{\prime}$ cannot vanish identically. This implies that radius of curvature of $C$ vanishes identically and $C$ reduces to a point. The streamlines are concentric circles with this point as centre.

In case of aligned flows (55) becomes

$$
\partial H / \partial \nu=0
$$

implying that $H=F(\xi)$, where $F$ is an arbitrary function of $\xi$. From (53), in this case 
we obtain the condition

$$
\begin{aligned}
3 \eta \psi^{\prime} \sigma^{\prime 2}+\eta \psi^{\prime} \sigma^{\prime \prime}(\xi-\sigma)+\left(\eta \psi^{\prime}-\sigma^{\prime} \psi^{\prime 2}+\mu F(\xi) \sigma^{2}\right)(\xi-\sigma)^{2}-\eta \psi^{\prime \prime}(\xi-\sigma)^{3} \\
+2 \eta \psi^{\prime \prime \prime}(\xi-\sigma)^{4}+\eta \psi^{(i v)}(\xi-\sigma)^{5} \equiv 0 .
\end{aligned}
$$

By the same argument as used carlier, we see that $\sigma^{\prime} \equiv 0$ and streamlines are concentric circles. Therefore, we have:

Theorem 3: If the streamlines in the fluid flow under study are involutes of some curve $C$, then $C$ must be a point and the streamlines are circles concentric at this point.

6. Isometric net. In this section we study those flows in which streamlines and their orthogonal trajectories coincide with the curves in an isometric net [4]. Martin [1] has studied this problem for non-MHD flows.

Let

$$
z=x+i y=z(\zeta)
$$

be an analytic function of $\zeta=\xi+i \sigma$. The curves $\xi=$ constant, $\sigma=$ constant in the $z$-plane (physical plane) form an isometric net. We want to determine all flows for which

$$
\phi=\phi(\xi), \quad \psi=\psi(\sigma),
$$

the functions $\phi(\xi), \psi(\sigma)$ being at our disposal.

The squared element of arc length in this $(\xi, \sigma)$ net is

$$
d s^{2}=\lambda(\xi, \sigma)\left[d \xi^{2}+d \sigma^{2}\right]
$$

$\lambda=\left|z^{\prime}(\zeta)\right|^{2}$. Using (60) in (16) and comparing with (61), we get

$$
E=\frac{\lambda(\xi, \sigma)}{\left[\phi^{\prime}(\xi)\right]^{2}}, \quad F=0, \quad G=\frac{\lambda(\xi, \sigma)}{\left[\psi^{\prime}(\sigma)\right]^{2}} .
$$

Substituting for $E, F, G$ from (62) and using (60) to introduce $\xi, \sigma$ as independent variables in the Gauss equation (20), we get

$$
\frac{1}{\phi^{\prime} \psi^{\prime}}\left(\frac{\partial^{2} l}{\partial \xi^{2}}+\frac{\partial^{2} l}{\partial \sigma^{2}}\right)=0
$$

where $l=\ln \lambda$, which merely confirms that $\ln \lambda$ is a harmonic function.

Noting that $\partial(x, y) / \partial(\xi, \sigma)=\lambda$, from (60), we have

$$
J=\partial(x, y) / \partial(\phi, \psi)=\lambda / \phi^{\prime} \psi^{\prime}
$$

Using (62), (64) in (32), we get

$$
z=\int \exp \left(\frac{1}{2} l+i \alpha\right) d \zeta
$$

where

$$
\alpha=\frac{1}{2} \int\left(-\frac{\partial l}{\partial \sigma} d \xi+\frac{\partial l}{\partial \xi} d \sigma\right)
$$

choosing $J>0$. If $m$ denotes a harmonic function conjugate to $l$, then $\alpha=\alpha_{0}+m / 2$ where $\alpha_{0}$ is an arbitrary constant and $z$ is given by 


$$
z=\int \exp \left(\frac{1}{2}(l+i m)\right) d \zeta
$$

Substituting for $E, F, G$ from (62) in system (34), we obtain

$$
\begin{gathered}
\eta\left[\frac{\partial^{2} \omega}{\partial \xi^{2}}+\frac{\partial^{2} \omega}{\partial \sigma^{2}}\right]-\psi^{\prime} \frac{\partial \omega}{\partial \xi}+\mu\left[\frac{\partial}{\partial \xi}(Q H \sqrt{ } \lambda) \cos \theta+\frac{\partial}{\partial \sigma}(Q H \sqrt{ } \lambda) \sin \theta\right]=0 \\
\omega=-\psi^{\prime \prime} / \rho \lambda \\
\frac{\partial H}{\partial \xi} \cos \theta+\frac{\partial H}{\partial \sigma} \sin \theta+\frac{1}{2} H \sin \theta \frac{\partial l}{\partial \sigma}+\frac{1}{2} H \cos \theta \frac{\partial l}{\partial \xi}=0 \\
\sqrt{ } \lambda Q=\frac{\partial H}{\partial \xi} \sin \theta-\frac{\partial H}{\partial \sigma} \cos \theta-\frac{1}{2} H \cos \theta \frac{\partial l}{\partial \sigma}+\frac{1}{2} H \sin \theta \frac{\partial l}{\partial \xi}
\end{gathered}
$$

We consider the aligned and non-aligned flows separately. In the case of the aligned flows, (68) becomes

$$
(\partial H / \partial \xi)+\frac{1}{2} H(\partial l / \partial \xi)=0
$$

which implies that

$$
\sqrt{ } \lambda H=F(\sigma)
$$

where $F(\sigma)$ is an arbitrary function of $\sigma$. On elimination of $\omega, Q$ and $H$ from (66), we obtain

$$
\left(\frac{\partial l}{\partial \xi}\right)^{2}+\left(\frac{\partial l}{\partial \sigma}\right)^{2}-\frac{2 \psi^{\prime \prime \prime}}{\psi^{\prime \prime}} \frac{\partial l}{\partial \sigma}+\frac{1}{\eta}\left(\psi^{\prime} \frac{\mu}{\psi^{\prime \prime}} F^{\prime}(\sigma) F(\sigma)\right) \frac{\partial l}{\partial \xi}+\frac{\psi^{(i v)}}{\psi^{\prime \prime}}=0
$$

provided that $\omega \neq 0$.

If we set

$$
\begin{aligned}
& \alpha=\frac{\partial l}{\partial \xi}, \quad \beta=-\frac{\partial l}{\partial \sigma}, \quad a=-\frac{1}{2 \eta}\left[\rho \psi^{\prime}-\frac{\mu}{\psi^{\prime \prime}} F^{\prime \prime}(\sigma) F(\sigma)\right], \\
& b=-\frac{\psi^{\prime \prime \prime}}{\psi^{\prime \prime}}, \quad c=\frac{\psi^{(i v)}}{\psi^{\prime \prime}},
\end{aligned}
$$

$\alpha+i \beta$ is an analytic function of $\zeta$ and (71) becomes

$$
\alpha^{2}+\beta^{2}-2 a \alpha-2 b \beta+c=0
$$

where $a=a(\sigma), b=b(\sigma), c=c(\sigma)$.

Martin [1] has shown that as a consequence of (73) $\alpha, \beta$ must be constant. In view of this we take $\alpha, \beta$ to be constants $\alpha_{0}, \beta_{0}$ in (72) and obtain

$$
l=\alpha_{0} \xi-\beta_{0} \sigma+\nu_{0}, \quad m=\beta_{0} \xi+\alpha_{0} \sigma+\delta_{0}
$$

where $\nu_{0}, \delta_{0}$ denote arbitrary constants.

From (6.)), we get

$$
z=C \int \exp (A \zeta) d \zeta
$$


where $A=\frac{1}{2}\left(\alpha_{0}+i \beta_{0}\right)$ and $C=\exp \left(\frac{1}{2}\left(\nu_{0}+i \delta_{0}\right)\right)$, so that

$$
\begin{aligned}
z & =z_{11}+C \zeta & \text { if } & A=0 \\
& =z_{n}+\frac{C}{A} \exp (A \zeta) & \text { if } & A \neq 0
\end{aligned}
$$

where $z_{0}$ is an arbitrary constant.

Since the streamlines are the transforms of the lines $\sigma=$ constant, they are restricted to (i) parallel lines if $A=0$, (ii) concurrent lines if $\alpha_{0} \neq 0, \beta_{0}=0$, (iii) concentric circles if $\alpha_{0}=0, \beta_{0} \neq 0$, (iv) logarithmic spirals if $\alpha_{01} \neq 0, \beta_{0} \neq 0$.

Now considering the case of non-aligned flows, from (66)-(69) and making use of (21), we get

$$
-\psi^{\prime \prime}\left[\left(\frac{\partial l}{\partial \xi}\right)^{2}+\left(\frac{\partial l}{\partial \sigma}\right)^{2}\right]+2 \psi^{\prime \prime \prime} \frac{\partial l}{\partial \sigma}-\psi^{(i v)}-\frac{1}{\eta} \psi^{\prime} \psi^{\prime \prime} \frac{\partial l}{\partial \xi}-\frac{l^{2} u}{\eta \sin ^{2}}-\frac{\psi^{\prime \prime}}{\theta} \psi^{\prime 3} \lambda^{2} \frac{\partial l}{\partial \xi}=0 .
$$

From (21), (62) and (68), we get

$$
\left(\psi^{\prime \prime} / \psi^{\prime}\right)=\cot \theta(\partial l / \partial \xi)+(\partial l / \partial \sigma)
$$

which, on differentiation with respect to $\xi$, gives

$$
\left(\partial^{2} l / \partial \xi \partial \sigma\right)+\cot \theta\left(\partial^{2} l / \partial \xi^{2}\right)=0 .
$$

Noting that $l$ is a harmonic function, we get

$$
\frac{\partial}{\partial \sigma}\left[\frac{\partial l}{\partial \xi}-\cot \theta \frac{\partial l}{\partial \sigma}\right]=0 .
$$

From (76) and (77), we conclude that

$$
\begin{aligned}
& \frac{\partial l}{\partial \xi} \cos \theta+\frac{\partial l}{\partial \sigma} \sin \theta=A \sigma+B \\
& \frac{\partial l}{\partial \xi} \sin \theta-\frac{\partial l}{\partial \sigma} \cos \theta=-A \xi+C
\end{aligned}
$$

and

$$
\psi^{\prime}=a \exp \left[\left(\frac{1}{2} A \sigma^{2}+B \sigma\right) \operatorname{cosec} \theta\right], \quad a \neq 0,
$$

$A, B, C$ being arbitrary constants.

As a result of (78), we obtain

$l=\frac{1}{2} A \sin \theta \sigma^{2}+A \cos \theta \xi \sigma-\frac{1}{2} A \sin \theta \xi^{2}+(B \cos \theta+C \sin \theta) \xi$

$$
+(B \sin \theta-C \cos \theta) \sigma+D_{1}
$$

and

$$
\begin{aligned}
m=\frac{1}{2} A \cos \theta \sigma^{2}-A \sin \theta \xi \sigma-\frac{1}{2} A \cos \theta \xi^{2}- & (B \sin \theta-C \cos \theta) \xi \\
& +(B \cos \theta+C \sin \theta) \sigma+D_{2}
\end{aligned}
$$

where $D_{1}, D_{2}$ are arbitrary constants. From (75), (79) and (80), we get 


$$
\begin{aligned}
& (A \sigma+B)^{3}+(-A \xi+C)^{2}(A \sigma+B)-2\left[(A \sigma+B)^{2} \operatorname{cosec} \theta+A\right](A \sigma \sin \theta+A \xi \cos \theta \\
& +B \sin \theta-C \cos \theta)+(A \sigma+B)\left[(A \sigma+B)^{2} \operatorname{cosec} \theta+3 A\right] \operatorname{cosec} \theta+\frac{\rho}{\eta} a(A \sigma+B) \\
& \quad \cdot(A \sigma \cos \theta-A \xi \sin \theta+B \cos \theta+C \sin \theta) \exp \left[\left(\frac{1}{2} A \sigma^{2}+B \sigma\right) \operatorname{cosec} \theta\right] \\
& +\frac{\mu k^{2}}{a^{3} \eta \sin ^{2} \theta}(A \sigma+B)(A \sigma \cos \theta-A \xi \sin \theta+B \cos \theta+C \sin \theta) \\
& \quad \cdot \exp \left[A\left(\sin \theta-\frac{3}{2} \operatorname{cosec} \theta\right) \sigma^{2}-A \xi^{2} \sin \theta+2 A \xi \sigma \cos \theta\right. \\
& \left.+2(B \cos \theta+C \sin \theta) \xi+(2 B \sin \theta-2 C \cos \theta-3 B \operatorname{cosec} \theta) \sigma+2 D_{1}\right] \equiv 0
\end{aligned}
$$

For the relation (82) to hold identically, $A=0$ and either

$$
B=0 \text { or } B \cos \theta+C \sin \theta=0 .
$$

From (65), we get

$$
\begin{aligned}
z & =z_{0}+D \zeta \\
& =z_{0}-\frac{2 D}{i(B \sin \theta-C \cos \theta)} \exp \left(-\frac{i}{2}(B \sin \theta-C \cos \theta) \zeta\right. \\
& \text { if } B \cos \theta+C \sin \theta=0 \text { and } B \sin \theta-C \cos \theta \neq 0
\end{aligned}
$$$$
\text { if } B=0 \text { and } C \neq 0 \text {. }
$$

where $D=\exp \left(\frac{1}{2}\left(D_{1}+i D_{2}\right)\right)$ and $z_{0}$ is an arbitrary constant.

As the streamlines are the transforms of the lines $\sigma=$ constant, they are restricted to (i) parallel lines if $B=C=0$, (ii) concurrent lines if $B=0, C \neq 0$, and $\theta= \pm \pi / 2$, (iii) concentric circles if $B \sin \theta-C \cos \theta \neq 0$ and $B \cos \theta+C \sin \theta=0$, (iv) logarithmic spirals if $\theta \neq \pm \pi / 2$ and $B=0, C \neq 0$.

Summing up, we have:

Theorem 4. If the streamlines and their orthogonal trajectories coincide with the curves in isometric net then the streamlines are restricted to (i) parallel straight lines, (ii) concurrent lines, (iii) concentric circles, (iv) logarithmic spirals if $\theta \neq \pm \pi / 2$.

In particular, in case of orthogonal flows we have:

Corollary: If the streamlines and magnetic lines coincide with the curves in an isometric net, then the streamlines must be (i) parallel straight lines, (ii) concurrent lines or (iii) concentric circles.

\section{REFERENCES}

[1] M. H. Martin, The flow of a viscous fluid. I, Arch. Rat. Mech. Anal. 41, 266-286 (1971)

[2] V. I. Nath and O. P. Chandna, On plane viscous magnetohydrodynamic flows, Quart. Appl. Math. 31, 351-362 (1973)

[3] G. Hamel, Spiralförmige Bewegungen Zäher Flüssigkeiten. Jber. Dtsch. Math-Ver. 25, 34-60 (1916)

[4] C. E. Weathernburn, Differential geometry of three dimensions, Cambridge, 1939 